\title{
5 Ergebnisse und Zusammenfassung
}

\subsection{An der Produktion und Rezeption beteiligte Akteure}

\subsubsection{Leser und Betrachter}

Die Personen und Institutionen, die an der Anbringung von Inschriften im öffentlichen Raum beteiligt waren, unterscheiden sich je nach Inschriftengattung. Rezipiert wurden die Inschriften dagegen von allen, die sich im Raum der Stadt bewegten - ob es sich nun um literate Leser oder illiterate Betrachter handelte, um solche, die gezielt nach Nachrichten von Freunden suchten, oder solche, die die Inschriften nur unbewusst wahrnahmen, ohne sie zu lesen. Aber wer sind die Schreiber und Auftraggeber? Welche Adressatenkreise wurden gezielt angesprochen und was bedeutete dies für andere Personen, die sich im Umfeld aufhielten?

Für die Steininschriften ist hinlänglich bekannt, dass sie nach umfangreicher Planung entstanden. Sie waren meist in Monumente oder Gebäude eingebunden und inhaltlich sowohl darauf wie auf bestimmte Konventionen und Regeln abgestimmt. Und auch das Herstellen der eigentlichen Inschrift erforderte es, dass der Wortlaut vorher festgelegt, dass im Idealfall die Verteilung der Buchstaben auf die Zeilen geplant und auch die nachfolgenden Arbeitsschritte korrekt ausgeführt wurden.

Sowohl für die Dipinti als auch für die Graffiti geht aber aus den Schriftquellen wie auch aus den Befunden hervor, dass sie ebenfalls geplant wurden und dass unterschiedliche Voraussetzungen wie die Verfügbarkeit von Materialien, Werkzeugen und Kenntnissen erfüllt sein mussten. Gerade bei den Graffiti mag das verwundern, da sie in der Regel als spontan entstandene oder gar deviante Äußerungen von Randgruppen verstanden werden. Es hat sich jedoch gezeigt, dass auch hier nicht jedes Objekt als Schreibgerät geeignet war, dass das Schreiben an der Wand einige Übung erforderte und dass viele Schreiber die Kursivschrift beherrschten. Die Anbringungsorte zeugen davon, dass die Schreiber sehr wohl unterschieden, ob sie eine bestimmte Person gezielt ansprechen wollten, indem sie etwa die Inschrift unmittelbar neben einer Haustür, in einem Grab oder in der Nähe eines bestimmten Geschäftes anbrachten, oder aber die Aufmerksamkeit einer breiteren Leser- und Betrachterschaft erregen wollten und die Inschrift gut sichtbar und hervorgehoben platzierten. Darüber hinaus ist bei Graffiti auch zu beobachten, dass sich die Schreiber nicht in jedem Fall an möglichen Adressaten orientierten, sondern mitunter auch der Akt des Schreibens oder Zeichnens im Vordergrund stand. Auch die Inhalte zeigen, dass viele Texte vorformuliert gewesen sein müssen, dass Bekanntes aufgegriffen oder auch bestimmte Inhalte im Vorfeld recherchiert werden mussten.

Was die Adressaten betrifft, richteten sich alle textuellen Inschriften natürlich primär an lesekundige Personen. Auch die hier beschriebenen Spezifika der pompejanischen Inschriften erlauben es nicht einfach, die Frage nach der Literalität mit „,ja“, 
„nein“ oder einer Prozentangabe zu beantworten. Die verwendeten nexus und Abkürzungen können nicht einfach gelesen werden, sondern erfordern Kenntnisse über das politische System der Stadt, Namen von Individuen und bestimmte Gepflogenheiten im Umgang mit Schrift. Das „Lesen“ war daher eine Konstruktionsleistung, die über das Entziffern von Buchstaben und das Erkennen syntaktischer Strukturen hinausging. Beispiele dafür sind die verschiedenen Abkürzungen für Personennamen, Ämter und Anmerkungen wie ovf, scr oder rog in den programmata. ${ }^{780}$ Doch auch Aspekte wie die Größe, die Farbe, die ordinatio und die Buchstabenformen, die scheinbar nicht „gelesen“ werden können, trugen zur inhaltlichen Rezeption bei. Die materiale Form ist nicht nur Hülle für einen Ausdruck, ${ }^{781}$ sondern die Inschriften sind überhaupt nur als materiale Form rezipierbar. Die Materialität der Inschriften hat großteils keine Entsprechung in der Lautsprache, ${ }^{782}$ sodass bei der Rezeption nur zum Teil an Lesen oder sogar lautes Lesen zu denken ist. Bei Inschriften, die viele Abkürzungen beinhalten, wäre zum Beispiel eher an „Ergänzen“ zu denken und die Hervorhebung bestimmter Textteile mit verschiedenen Farben könnte allenfalls mit einem Kommentar wiedergegeben werden. ${ }^{783}$ Jede der drei Inschriftengattungen brachte unterschiedliche Herausforderungen für die Rezipienten mit, die sich mit den Inschriften auseinandersetzten. Aber wie wirkte der beschriebene Raum auf solche Passanten, die sich nicht mit den Graffiti und Dipinti auseinandersetzten, sondern - wie Plutarch es fordert - daran vorbeigingen? Wer waren die Schreiber und wie agierten sie? Wie gingen sie mit den Fassaden als Beschreibflächen um und was sagt dies über die Bedeutung und die Funktion der Wandinschriften aus?

\subsubsection{Schreibertrupps, Malwerkzeuge, Arbeitsschritte}

Während einerseits für Steininschriften die Chaîne opératoire gut erforscht ist und andererseits bei Graffiti Auftraggeber und Ausführende identisch waren, stellt sich hinsichtlich der Anbringung der Dipinti die Frage, wer an deren Anbringung praktisch beteiligt war. Handelte es sich um professionell organisierte Einzelpersonen oder Teams? Agierten Sklaven im Auftrag ihrer Herren? Welche Alternativen sind denkbar? Aus den Inschriften erfahren wir sowohl Namen als auch Hinweise auf konkrete Tätigkeiten und die Organisation der Arbeiten.

Die programmata, edicta munerum und die Grüße unter den Dipinti sprechen selbst eindeutig dafür, dass diese von professionell agierenden Einzelpersonen und Gruppen angebracht wurden, die über Erfahrung und Spezialkenntnisse verfügten

780 Dies begegnet aber auch in den Graffiti, z. B. in CIL IV 8215: quos L V P amat valeant, bei denen heutigen Lesern unbekannt ist, für welche Namen die Abkürzung $L V P$ steht.

781 So Foucault 1969, 131-138.

782 Vgl. dazu Krämer 2006, 76-78.

783 Vgl. Susini 1989, 297. 
und für ihre Arbeiten bezahlt wurden. Dies erweist sich zum einen an der erstklassigen graphischen Gestaltung und zum anderen an den entsprechenden Vermerken mit Namensnennungen. Dabei wird jedoch nie das Nomen scriptor, sondern immer die Abkürzung scr oder eine Form von scribere (Abb. 55) verwendet. ${ }^{784}$ Die Schreiber scheinen meist in Gruppen mit unterschiedlicher Besetzung und Aufgabenverteilung gearbeitet zu haben. Neben dem Vermerk scr finden sich nämlich auch Hinweise auf andere Tätigkeiten. Eine Person war dafür zuständig, erforderlichenfalls die benötigte Fläche mit Kalkmilch weiß anzustreichen und wurde mit dealbator oder dealbare bezeichnet. ${ }^{785}$ Außerdem sind die Begriffe lanternarius und adstans belegt. Allerdings kommen sie beide je nur einmal vor, sodass insbesondere bei letzterem fraglich ist, wie ernst eine so vage gehaltene Aufgabenbeschreibung gemeint sein kann. ${ }^{786}$ Daneben war möglicherweise eine weitere Person beteiligt, die eine Leiter trug und anstellte. ${ }^{787}$

Indirekt bestätigt auch der Dipinto des Mustius, dass normalerweise in Teams gearbeitet wurde: M(arcum) Pupium Rufum // IIvir(um) i(ure) d(icundo) dignum r(ei) p(ublicae) o(ro) f(aciatis) / Mustius fullo facit / et dealbat scr(ipsit) unicus / s[ine] reliq(uis) sodalib(us) non(is). ${ }^{788}$ Explizit wird einerseits darauf hingewiesen, welchen Beruf der Schreiber hauptamtlich ausübte und andererseits dass er allein arbeitete. Diese ausführliche Form des Autorenvermerks ist unter den Dipinti singulär und bestätigt daher eher die Annahme, dass in der Regel spezialisierte Schreiberteams die Schriftzüge malten. Weitere eindeutige Beispiele, in denen die Schreiber einen Beruf nennen, sind bisher nicht bekannt. Daher kann zwar nicht ausgeschlossen, dass die Schreibertrupps ihren Lebensunterhalt auch durch eine andere Tätigkeit bestritten, doch es deutet nichts darauf hin, dass dies die Regel war. ${ }^{789}$ Wahrscheinlich konnten sie jedoch ihre Expertise auch anderweitig einsetzen: Gerade auch die Mitarbeit an Steininschriften oder die Erstellung heute nicht mehr erhaltener Schriftzeugnisse, wie der hölzernen tabulae dealbatae, die häufiger neu angefertigt und beschrieben werden mussten, oder die Anfertigung von Ladenschildern könnten ebenso zu den Aufgaben bzw. Leistungen von professionellen Schreibern gehört haben. Und selbst wenn man annimmt, dass ein Teil der Schreiber sich seinen Lebensunterhalt durch verschiedene Tätigkeiten verdiente, ändert dies nichts daran, dass für die Anbringung von Dipinti spezifische Fertigkeiten vonnöten waren.

Die Anbringungsstellen der Dipinti oder ihre Inhalte lassen keine Aussage darüber zu, ob sie prinzipiell tags oder nachts arbeiteten. In den nur spärlich beleuchteten

784 De Marchi 1916, 65-66; Franklin 1980, 24; Sabbatini Tumolesi 1980, 122-123; Weber 1982, 79; Mouritsen 1988, 31; Staccioli 1992, 50; Donati 1998, 101; Chiavia 2002, 86; Wallace 2005, XI. Eine Reihe namentlich bekannter Schreiber findet sich bei Franklin 1978, 58-73.

785 Z. B. CIL IV 222 und 1190.

786 CIL IV 1190. 7621. Vgl. dazu: Franklin 1986, 322; Ciprotti 1975, 274-275.

$787 \mathrm{Zu}$ den verschiedenen Aufgaben und der Zusammensetzung der Truppe: De Marchi 1916, 67; Magaldi 1930, 62-65; Franklin 1978, 58-73; Weber 1982, 80; Staccioli 1992, 51; Chiavia 2002, 89-90.

788 CIL IV 3529.

789 Magaldi 1930, 53-55; so auch: Sabbatini Tumolesi 1980, 123 Anm. 36. 


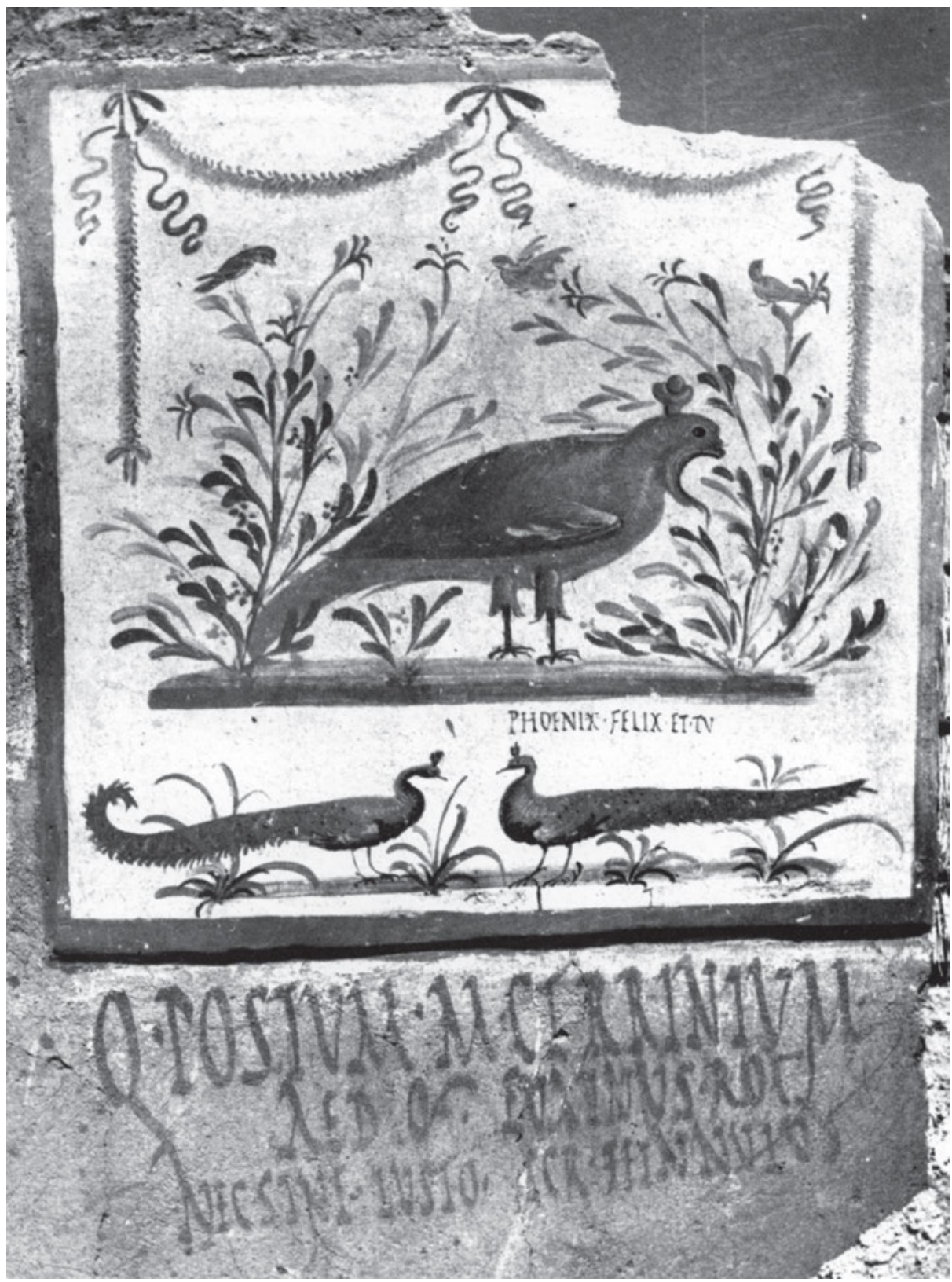

Abb.55: CIL IV 9850. 9851 von Eingang I 11, 11. @ Su concessione del Ministero per i Beni e le Attività Culturali e per il Turismo - Parco archeologico di Pompei. Jegliche weitere Reproduktion oder Duplikation ist untersagt. 


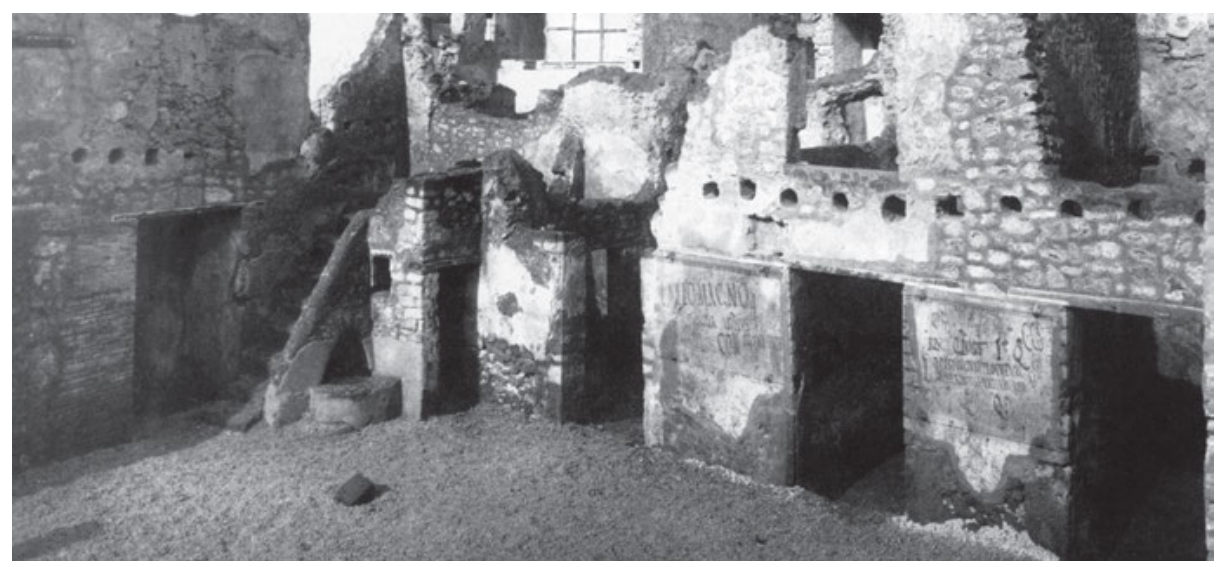

Abb.56: Dipinti im Hof von I7, 16: CIL IV 7243-7246. ( Su concessione del Ministero per i Beni e le Attività Culturali e per il Turismo - Parco archeologico di Pompei. Jegliche weitere Reproduktion oder Duplikation ist untersagt.

Straßen der Stadt war es nachts deutlich schwieriger, eine Ankündigung auf die Wand zu pinseln. Doch selbst wenn eine gute Beleuchtung unerlässlich war, um einen ästhetisch hochklassigen Dipinto zu malen, sahen sich die Maler tagsüber einer Vielzahl anderer Hindernisse gegenüber, die unter Umständen doch dazu führen konnten, dass an manchen Orten nachts gearbeitet wurde. Viele Dipinti befinden sich weit über Kopfhöhe, sodass sie nicht ohne eine Leiter gemalt werden konnten. Um diese anzustellen, brauchte der Schreiber aber zumindest den Bürgersteig und bei schmalen Straßen auch die Fahrbahn. Beide waren tagsüber von Fußgängern und Tieren bevölkert und auf den Bürgersteigen wurden teilweise die Verkaufsflächen der Läden erweitert. Daher war es schlichtweg nicht überall möglich, zu jeder Tageszeit zu arbeiten.

Werkstätten, Ladengeschäfte oder Niederlassungen von Schreibern konnten bislang nicht eindeutig identifiziert werden. Eine mögliche Niederlassung von Schreibern wurde in dem Haus I 7, 16 erkannt (Abb. 56). Dafür spricht, dass dort mehrere auch anderweitig bekannte Schreibernamen auftauchen, dass einzelne Buchstaben ohne Zusammenhang geschrieben wurden und dass diese Flächen im Innenbereich eines Hauses lagen, wodurch sie keine breite Werbewirkung entfalten konnten. ${ }^{790}$ Bei dem Gebäude handelt es sich um ein wenigstens zweigeschossiges Wohnhaus, dessen Erdgeschossräume sich an der Nordseite eines großen Hofes oder Gartens erstrecken. Im Hof befand sich ein gemauertes Sommertriclinium. ${ }^{791}$ Das Haus wurde mit Sicherheit nicht als Werkstatt gebaut. Dennoch ist es denkbar, dass es zur Zeit des Vesuvausbruches nicht mehr als repräsentatives Wohnhaus, sondern als Geschäftsniederlassung diente. Eindeutige Belege, wie etwa Funde von Werkzeugen oder Materialien fehlen jedoch.

790 Della Corte 1926, 650; Franklin 1978, 55; Staccioli 1992, 50.

791 Jashemski 1993, 41. 
Auch die Gestaltung der Wandinschriften liefert Informationen über die Vorgehensweise der Schreiber. Die professionellen Schreiber müssen, wenn sie edicta munerum, programmata, Grüße, Mietanzeigen oder andere planvoll angelegte Dipinti wie die alba malten, inhaltlichen Vorgaben gefolgt sein. Je nach Art der Inschrift waren diese mehr oder weniger detailliert. Bei edicta munerum oder Mietanzeigen, bei denen der Inhalt sehr spezifisch und im Detail unterschiedlich war, brauchten sie mehr Angaben als bei programmata, bei denen sich die notwendigen Informationen nahezu auf den Namen und das Amt beschränkten. In der Gestaltung und der Formulierung konnten sich die Schreiber dieser Dipinti daher einiger Freiheiten bedienen oder waren möglicherweise auch vom Auftraggeber dazu angehalten, von verschiedenen Abkürzungen, Farben, Schriften und Größenverhältnissen Gebrauch zu machen. Varianz findet sich bei den rogatores. Hier sind auch uneinheitliche oder sogar außergewöhnliche Formulierungen zu finden. Die Gestaltung war zudem immer auch davon abhängig, wie viel Platz zur Verfügung stand und wie ein Dipinto in einem bestimmten räumlichen Kontext zur Geltung gebracht werden konnte. ${ }^{792}$

Nur wenige Dipinti wurden offensichtlich von Personen geschrieben, die keine oder wenig Übung im Umgang darin hatten, auf Wände zu schreiben. Dies sind einige programmata für Aulus Suettius Verus ${ }^{793}$ und diejenigen des Fufidius Successus. ${ }^{794}$ Bei beiden ist zu beobachten, dass sich die Schreiber um ein ordentliches Schriftbild bemühten, ohne aber die üblicherweise verwendeten Buchstabenformen der actuaria zu treffen. In diesen Fällen ist davon auszugehen, dass die Schreiber in Eigenregie arbeiteten, sich jedoch im Wortlaut an den professionell verfertigten programmata orientierten. Es kann ausgeschlossen werden, dass finanzielle Gründe die Suettii daran hinderten, professionelle Schreiber zu engagieren, da mindestens zwei edicta munerum belegen, dass Aulus Suettius Certus als aedilis Gladiatorenspiele veranstaltete. ${ }^{795}$ Daher liegt die Vermutung näher, dass hier tatsächlich eine Gruppe von Anhängern (ähnlich wie der erwähnte Fufidius Successus), die mit dem üblichen Aussehen und Inhalt vertraut, im Malen aber weniger geübt waren, selbst tätig wurde. ${ }^{796}$ Ebenso wäre es aber auch möglich, dass die Kandidaten ihre eigenen Sklaven damit beauftragt hatten, die Dipinti anzubringen. Bei anderen Inschriften, wie den cacatorInschriften ist hingegen anzunehmen, dass sie von Anwohnern angebracht wurden, ohne dass sie inhaltlich von offiziellen Bekanntmachungen abhängig waren.

792 Magaldi 1930, 71-73; De Marchi 1916, 68-71; Sabbatini Tumolesi 1980, 122; Staccioli 1992, 53; Chiavia 2002, 76-77.

793 Für Aulus Suettius Verus: CIL IV 1137. 7541. 7748. 7751. 7789. 7813. 7828. 7926 und 7939; für Aulus Suettius Certus: CIL IV 7660 (gemeinsames programma für Verus und Certus). 7700. 7752. 7823 . 7823b. und 7834. Zu CIL IV 7691: Spinazzola 1917, 260.

794 Vgl. Kapitel 4.1.3.2.

795 CIL IV 1189 und 1190; Sabbatini Tumolesi 1980, 51-53. In CIL IV 1190 wird zudem eine ganze Reihe von Personen genannt, die handwerklich an der Anfertigung dieses Dipinto beteiligt waren.

796 Vgl. Fioretti 2012, 419 Anm. 34. 


\subsubsection{Alles erlaubt? Schreiben auf den Fassaden von Wohnhäusern}

Bei Steininschriften wurde oft klar formuliert, mit welcher Berechtigung oder vor welchem institutionellen Hintergrund das Monument oder Bauwerk, mit dem sie verbunden waren, errichtet und gestaltet worden war. Diese befinden sich meist im öffentlichen Raum und es ist bekannt, dass dort Regeln und Gesetze die Errichtung von Denkmälern und Grabstätten regulierten. Bei Graffiti und Dipinti, die häufig oder meist an Fassaden von in Privatbesitz befindlichen Häusern angebracht wurden, bleibt zu fragen, ob deren Anbringung geduldet, erlaubt oder sogar erwünscht war. Dahinter steht einerseits die grundsätzliche Überlegung, welche Rechte und Pflichten die Anwohner hinsichtlich der Gestaltung ihrer Fassaden hatten, und andererseits die Frage danach, wie die Maler, Auftraggeber und Anwohner im jeweiligen Fall miteinander interagierten, wenn ein neuer Dipinto angebracht werden sollte. Auf welcher Basis und unter welchen Bedingungen wurden Graffiti und Dipinti an die Fassaden geschrieben, gezeichnet und gemalt?

Die Annahme, dass das Beschreiben von Wänden illegal oder als ordnungswidrig aufgefasst wurde, ist nicht in antiken Texten oder Befunden begründet, sondern wie oben ausgeführt in Sichtweisen und Bewertungen von modernen Graffiti und Wandaufschriften sowie dem heutigen stark regulierten und kommerzialisierten Umgang mit Werbung und besonders Wahlwerbung im öffentlichen Raum. ${ }^{797}$ Die antiken Texte und Inschriften deuten nicht darauf hin, dass ein generelles Verbot zur Anbringung von Inschriften an Fassaden von Privathäusern oder Gräbern bestand. Zwar weisen manche Schriftquellen, Graffiti oder Steininschriften darauf hin, dass die Texte mit Missfallen betrachtet wurden oder an bestimmten Stellen nicht erwünscht waren. ${ }^{798}$ Gerade weil diese Urteile und Verbote individuell ausgesprochen wurden, ist aber anzunehmen, dass es im Regelfall nicht verboten war, Wände zu beschreiben. Darüber hinaus gibt es aber Hinweise, dass Absprachen mit den Eigentümern getroffen wurden, wenn es darum ging, großformatige programmata und edicta munerum anzubringen. ${ }^{799}$

Die Fassaden gehörten zum Haus und es ist offensichtlich, dass die Gestaltung dem jeweiligen Eigentümer oblag. Das ergibt sich zum einen aus erhaltenen Befunden, in denen jeweils an der Grenze zwischen zwei Grundstücken auch das

797 Je nach Bundesland und Kommune bestehen z. B. in Deutschland eigene Regelungen. In der Regel dürfen Wahlplakate an öffentlichen Straßen und Wegen ab 30 Tagen vor dem Ereignis aufgestellt werden und müssen innerhalb einer Woche danach wieder entfernt werden. Auch die zulässigen Orte sind explizit benannt. Die Anbringung an privaten Fassaden ist nicht gestattet, außer mit Genehmigung des Eigentümers: Vgl. dazu z. B. das Berliner Straßengesetz (BerlStrG) §11 Abs. 2a oder die Fachanweisung über die politische Werbung auf öffentlichen Wegen mit Werbeträgern der Hamburgischen Finanzbehörde 3.3, 3.4, 6.2 und 8.

798 So etwa Plut. de curiositate (520D-E) und Mart. 12,61,7-11; CIL IV 1904. 2461. 2487.

799 Vgl. Gesemann 1996, 177. Vgl. Staccioli 1992, 48-49. 
Gestaltungsschema wechselt. Zum anderen wird dies mit Bezug auf die Bürgersteige in dem auf der tabula heracleensis überlieferten Gesetzestext explizit formuliert ${ }^{800}$ und es ist anzunehmen, dass auch für die Fassaden ähnliche Regelungen bestanden. Die Frage ist nun, welches Interesse ein Anwohner daran haben konnte, dass seine Fassaden beschrieben wurden. Wenn er gesetzlich dazu gezwungen gewesen wäre, die Beschriftungen zu akzeptieren, hätte doch zumindest nach den Wahlen und auf den nicht dekorierten Flächen die Möglichkeit bestanden, unliebsame Texte wieder zu übermalen. Dies geschah aber offenbar in der Regel nicht.

Della Corte geht davon aus, dass es eine enge Beziehung zwischen den in den programmata als rogatores genannten Personen und den Inhabern der beschrifteten Häuser gab und knüpft daran Aussagen über die Bewohner der Häuser. ${ }^{801}$ Seiner Ansicht nach sorgten in der Regel die Bewohner selbst aktiv dafür oder befürworteten es zumindest, dass an ihrem Haus programmata angebracht wurden, die einen Mitbürger für ein städtisches Amt empfahlen. Dies kann jedoch nicht der einzige Weg gewesen sein, allein schon, weil die Namen oft an ganz unterschiedlichen Orten auftauchen und weil an den Häusern verschiedene Personen als rogatores gleich oft erwähnt werden. ${ }^{802}$ Gerade bei dicht beschriebenen Fassaden stellt sich daher die Frage, ob der Eigentümer möglicherweise Teilflächen explizit zur Verfügung stellte - sei es, um sich mit einem potentiellen zukünftigen Beamten gut zu stellen, aufgrund persönlicher Beziehungen oder möglicherweise sogar gegen Bezahlung. Bei den Dipinti und besonders bei den Fallbeispielen war aufgefallen, dass die Maler sich häufig an der Gestaltung der Fassaden orientierten (Abb.13. 14. 43). Dies hängt vermutlich vorrangig mit ästhetischen Überlegungen zusammen und mit der Absicht, der jeweiligen Inschrift zu Aufmerksamkeit zu verhelfen. Die Maler kamen so aber auch dem Eigentümer der Fassade entgegen, indem sie deren Gliederung und Gestaltung respektierten.

Daraus könnte man schließen, dass die Anwohner ihre Fassaden gegen Geld für die Beschriftung zur Verfügung stellten und dann jeweils eine bestimmte Fläche auswiesen, die die Schreiber nutzen konnten. Dies mag in Einzelfällen an besonders belebten und publikumsreichen Stellen der Fall gewesen sein. Allerdings gibt es zahlreiche Fälle, in denen die programmata und edicta munerum über die Grenze zwischen zwei Häusern hinwegreichen, was darauf hindeutet, dass keine Vergütung erbracht werden musste. ${ }^{803}$ Dies wiederum legt nahe, dass grundsätzlich keine Absprachen

800 Vgl. CIL I 593, Zeile 53-55: quoius ante aedificium semita in loco <publico > erit, is eam semitam eo aedificio perpetuo lapidibus perpetueis / integreis continentem constratam recte habeto arbitratu eius aed(ilis), quious in ea parte h(ac) l(ege) uiarum / procurat<io > erit. Zitiert nach: Crawford/Nicolet 1996, 365; s. a.: 360-362. Vgl. Pirson 1999, 65. Für Pompeji wurden bisher keine spezifischen Gesetzestexte bekannt.

801 Dies ist eine der Methoden, die er in „Case ed Abitanti“ anwendet: Della Corte 1926, 2-3.

802 Vgl. Mouritsen 1988, 18-19. Ebenfalls kritisch: Allison 2001, 54. 69.

803 Vgl. CIL IV 7993 an der Fassade von III 2, 1 und III 2, 2 oder das edictum muneris an den Gräber ES 9 und ES 11 in der Nekropole vor der Porta di Nocera: CIL IV 9983a. 
nötig waren. ${ }^{804}$ Die Anwohner hatten es offenbar in der Regel zu akzeptieren, dass Ihre Fassaden beschrieben wurde. Völlig frei gelassene Wände deuten jedoch darauf hin, dass es möglich war, dem zu widersprechen. Wie an den Nennungen der rogatores und anderer Unterstützer zu erkennen ist, waren besonders die programmata Teil eines komplexen Gefüges gegenseitiger Unterstützung und demonstrativer Zurschaustellung politischer Seilschaften. Dementsprechend kann es sich für den Anwohner, der sich gegen die Beschriftung seines Hauses verwahrte, möglicherweise negative Folgen gehabt haben, da er an dieser Praxis nicht teilnahm.

Auch bei den Graffiti deuten die Befunde und die Metatexte eher darauf hin, dass es geduldet, akzeptiert oder sogar erwünscht war, dass die Wände beschrieben wurden. Darauf deuten einerseits die vielen Namen hin, andererseits aber auch die Verteilung an den unterschiedlichsten Orten, z. B. auch in und an Geschäften, wo der Inhaber es zu den Öffnungszeiten leicht hätte verhindern können, dass ein Besucher seine Wand beschriftete. In den Metatexten kommt es zwar vor, dass ein Schreiber anonym bleibt oder heimlich und nachts arbeitet. Dies ist jedoch jeweils durch den Inhalt der Graffiti zu begründen. ${ }^{805}$

\subsection{Kommunikative Funktionen und Rezeption von Inschriften im öffentlichen Raum}

Die Frage nach den kommunikativen Funktionen der Inschriften zielt auf den Kern ihrer Bedeutung ab und trifft bezüglich der hier behandelten Inschriften doch das Ziel nur zum Teil. Ähnlich wie in der Sprechakttheorie wird in der Textlinguistik versucht, in den schriftlichen Äußerungen die Absichten des jeweiligen Urhebers (z. B. eines Briefschreibers) zu erkennen. Und in der Tat sind bei den Inschriften repräsentative, direktive, kommissive, expressive und deklarative Illokutionen festzustellen. ${ }^{806}$ In Bau-, Stifter- oder Grabinschriften werden Sachverhalte beschrieben, edicta munerum erläutern das Angebot zukünftiger Ankündigungen (repräsentativ); programmata fordern zur Wahl einer bestimmten Person auf, edicta munerum zum Besuch einer Veranstaltung und Preisangaben zum Kauf eines Produktes auf (direktiv); die cacatorInschriften warnen vor Konsequenzen und in manchen programmata wird deutlich, dass mit dem Amt auch Pflichten kamen (kommissiv); es gibt Grüße und Glückwünsche wie auch Ehrbezeugungen (expressiv); mit der Anbringung von Markierungen

804 Mouritsen nimmt an, dass die Anwohner keinerlei Einfluss auf die Beschriftung der Fassaden hatten: Mouritsen 1988, 58. 193 Anm. 211. Dagegen Viitanen/Nissin/Korhonen 2013, 73-74.

805 Lukian, Dial. Mer./Het. dial. 10,4 (14) und Plut. Caius Gracchus 17,9 (8). Vgl. Lohmann 2018, 10-11, und allgemein zur Akzeptanz von Graffiti im öffentlichen wie im privaten Raum: Baird/Taylor 2011, 3-4, Benefiel 2011, 20 und Langner 2001, 20.

806 Nach der Einteilung und Erläuterung von John R. Searle, angelehnt an John L. Austins „How to Do Things With Words“: Vgl. Searle 1976, 10-16. Austin 1962. 
werden Grenzen gesetzt und Verkaufsräume zugewiesen (deklarativ). ${ }^{807}$ Das Hauptaugenmerk bei der Frage nach den Funktionen liegt somit auf den mehr oder weniger bewussten Intentionen der Urhebers. Der Leser oder Hörer wird insofern berücksichtigt, als gefragt wird, ob dieser die Intention des Urhebers auf einer vorrangig sprachlichen Ebene versteht und welchen perlokutionären Effekt, der so vom Sprecher geplant gewesen sein kann oder auch nicht, eine Äußerung erzielte. ${ }^{808}$ Ausschlaggebend dafür, ob der Akt gelingt, ist es, dass der konventionale Rahmen eingehalten wird, dass also die notwendigen Vorkenntnisse beim Rezipienten vorhanden waren und dass der Urheber sich an Regeln und Gepflogenheiten hielt. Der springende Punkt ist, dass in der Sprechakttheorie Sprache nicht als Instrument zur Beschreibung verstanden wird, sondern dass durch Äußerungen Handlungen vollzogen werden. Sprache ist aber jeweils nur ein Teil des Ausdrucks.

Die Sprechakttheorie zielt nicht speziell auf Inschriften, wohl aber auch auf schriftliche Äußerungen ab. Im Bereich der Textlinguistik zeigt sich, dass sie für unterschiedliche schriftlich verfasste Textsorten gewinnbringend genutzt werden kann. ${ }^{809}$ Der Versuch einer Anwendung der Sprechakttheorie auf Inschriften kann daher dazu dienen, Besonderheiten dieser Form von Schriftlichkeit deutlicher hervortreten zu lassen. ${ }^{810}$

Sowohl bei Steininschriften als auch bei Dipinti gibt es nicht einen Urheber, sondern mehrere Instanzen, die jeweils unterschiedliche Interessen verfolgten. An der Formulierung und Anbringung einer Ehreninschrift waren städtische Institutionen, gegebenenfalls natürliche Personen, die das Monument finanzierten, der oder die Geehrten selbst und deren Familien beteiligt. Die programmata wurden auf Initiative des Kandidaten und der rogatores in Auftrag gegeben, ausgeführt wurden die Arbeiten von professionellen Schreibern. Alle verfolgten unterschiedliche, in der Regel aber miteinander vereinbare Ziele. Aber wessen Intention wäre hier vorrangig interessant? Bei Inschriften, die im öffentlichen Raum der Stadt angebracht waren, ist zudem von großer Bedeutung, dass sie dort über einen längeren Zeitraum präsent waren, nicht als Verdauerung einer mündlichen Rede, sondern als Schriftartefakte. ${ }^{811}$ Damit ist gemeint, dass im räumlichen Kontext mit anderen Artefakten, mit Menschen und Handlungen die sprachliche Äußerung nur ein Aspekt der Inschrift unter mehreren anderen war. Und andererseits änderte sich der Rahmen, in dem die Leser oder Betrachter mit der Inschrift konfrontiert waren, im Laufe der Zeit. Es kamen neue

807 Textlinguistische Analyse von Textfunktionen weitgehend parallel zur Sprechakttheorie in Brinker/Cölfen/Pappert 2014, 98-125 und Große 1976.

808 Vgl. Austin 1962, 101.

809 Brinker/Cölfen/Pappert 2014, 125-131. Zur Anwendung auf Inschriften und dem Bezug zur Materialität sowie zum Begriff des „Schriftaktes“ in diesem Kontext: Frese/Keil 2015, 633-638.

810 Hier kann nicht umfassend auf die Funktionen und die Entwicklung römischer Inschriften eingegangen werden. Vgl. dazu grundlegend: MacMullen 1982, 244-246; Meyer 1990, 74-78. Einen guten Überblick bietet: Beltrán Lloris 2015.

811 Ausführlich in Krämer 2003 und Krämer 2006. Jacques Derrida zur Funktion, Bedeutung und Merkmalen der Schrift und besonders ihrem Verhältnis zur Sprache: Derrida 1983, 29-32. 51-77. 
Inschriften und Monumente hinzu. Renovierungen wurden durchgeführt und neue Gebäude errichtet. Auch die Voraussetzungen und Konventionen seitens der Betrachter änderten sich. Wer konnte im Jahr 79 n. Chr. noch die oskische Schrift und Sprache verstehen? Wer kannte noch die Familien oder Individuen, die in augusteischer Zeit einflussreich gewesen waren? Wer konnte ein Zitat richtig einordnen? Welche neuen Bezüge kamen auf der anderen Seite hinzu? Wie veränderte etwa der Wechsel zur flavischen Dynastie den Blick auf Nero und dessen Namen? Dass diese Fragen aufkommen mochten, konnte zur Folge haben, dass die ursprünglichen Intentionen der beteiligten Urheber immer weniger verstanden wurden. Tatsächlich heißt es aber vor allem, dass diese eine immer geringere Bedeutung hatten, da sich die Umstände und Konventionen im Vergleich zur Entstehungszeit wandelten. Dass ein 15 Jahre altes programma ein Wahlaufruf war, hatte für den aktuellen Leser nicht mehr zur Folge, dass er sich zur Stimmabgabe aufgefordert sah, sondern vielmehr, dass er den damaligen Kandidaten oder seine Familie bestimmten sozialen Rollen und Schichten zuordnen und möglicherweise Verknüpfungen $\mathrm{zu}$ anderen, später entstandenen Inschriften herstellen konnte. Und auch wenn kaum jemand mehr die oskischen Texte las und daher ihre textuellen Inhalte nur schwierig rezipierbar waren, konnten sie doch für den Betrachter um 79 n. Chr. Zeugnisse der Vergangenheit der Stadt Pompeji oder ein Hinweis auf das Alter eines Hauses oder Stadtviertels sein. Solche neuen Bedeutungen ergeben sich aus dem Kontext, den Handlungen und der Konstruktionsleistung der jeweils aktuellen Betrachter. Genau dies hat sich auf allen Analyseebenen als konstitutiv für die Wand- und die Steininschriften erwiesen.

Bei den Graffiti zeigte sich, dass sich die Intentionen des Urhebers besonders stark auf die Rezeption der Inschriften auswirkten. Diese waren inhaltlich weniger stark formalisiert als Dipinti und Steininschriften und gaben somit den Urhebern verschiedene Möglichkeiten, ihre Wahrnehmung zu steuern: Durch die Anbringungsstelle, die Größe und Gestaltung und natürlich auch durch den textuellen Inhalt. Je näher ein Graffito etwa an einer Haustür, in der Nähe eines bestimmten Geschäftes oder auch an schwer sichtbaren Stellen angebracht wurde, desto eher ist damit zu rechnen, dass der Schreiber gezielt eine oder mehrere bestimmte Personen ansprechen wollte und auch eine Reaktion - sei es in schriftlicher oder anderer Form - erwartete bzw. einen bestimmten Effekt erzielen wollte. Bei anderen Inschriften und Zeichnungen, die an belebten Orten oder mitten auf einer Wand angebracht waren und darüber hinaus inhaltlich keinen Bezug auf einen bestimmbaren Adressaten nehmen, ist dagegen anzunehmen, dass es auch um den Akt des Schreibens und den Stolz auf das eigene Produkt ging, bzw. darum durch diese Inschrift die eigene Person und gegebenenfalls Aktivitäten im öffentlichen Raum sichtbar und präsent zu machen. ${ }^{812}$ Einerseits wurde somit durch die Anbringung im öffentlichen Raum eigentlich private oder nur

812 Vergleiche hierzu etwa die Befunde an der Fassade IX 9, a-g. Zur Bedeutung des Schreibvorganges für die Graffitischreiber siehe auch: Lohmann 2018, 76-79. 272-278. 
wenige Personen direkt betreffende Kommunikation öffentlich gemacht. Andererseits wurden durch das Schreiben auf den Wänden diese überhaupt erst zu einem Ort, der Aufmerksamkeit anzog und im Diskurs bestimmter Themen eine Rolle spielte. Die Schriftquellen und Metatexte zu den Graffiti weisen ebenfalls in diese Richtung. Bei einigen der dort zitierten Graffiti wird eine kommunikative Funktion mitgedacht, formuliert und versucht, die Inschrift möglichst so zu gestalten, dass diese erfüllt werden kann. Auch die gezielte Platzierung zur Ansprache von bestimmten Adressaten wird dort thematisiert. ${ }^{813}$ In anderen Quellen steht dagegen der öffentliche Charakter der Wände, die Breite an Themen und die hohe oder niedrige Relevanz für den jeweiligen, unbestimmt gedachten Betrachter im Vordergrund. ${ }^{814}$ Dies ist symptomatisch für den scheinbar ambivalenten Charakter der Graffiti im öffentlichen Raum. Es geht um Persönliches, zwischenmenschliche Beziehungen oder schlichtweg für die Öffentlichkeit eigentlich irrelevante Inhalte, die aber doch im öffentlichen Raum präsent waren und somit zwangsläufig Teil eines öffentlichen Diskurses wurden. Hierin lässt sich vielleicht die einzige gemeinsame kommunikative Funktion der hier behandelten Graffiti feststellen: die Erweiterung des eigenen Handlungsrahmens um die wenn auch mitunter sehr eingeschränkte öffentliche Sichtbarkeit und Wirksamkeit des Individuums.

Die Wirkung und Wahrnehmung der Dipinti konnten vielschichtig sein. Wenn auf der Ebene der lesenden und rein inhaltlichen Rezeption suggeriert wird, der Appell, jemanden zu wählen oder eine Veranstaltung zu besuchen, sei der unveränderliche Kern der Botschaft, hat sich doch besonders in den Fallbeispielen gezeigt, dass häufig das ,wie‘, ,wann' und ,wo' ausschlaggebender für die Rezeption war als der Text. Größe, Position, Kontext und Gestaltung bestimmten, wer, bei welcher Gelegenheit, wie schnell, mit welchen Vorannahmen und Erwartungen einen Wahlaufruf zur Kenntnis nahm. Abgesehen von Ihrer Einbettung in ihren räumlichen und zeitlichen Kontext sind die programmata aber auch in ihrem Kontext der Wahlen als politischem und sozialem Vorgang zu sehen. Es ist davon auszugehen, dass die Kandidaten offiziell auf anderem Wege nominiert wurden und dass die Urheber der programmata sich darauf verließen, dass die Bevölkerung Zugang zu diesen Informationen hatte. ${ }^{815}$ Dafür spricht auch, dass nie ein Jahr der Kandidatur genannt wird und dass in einigen programmata scheinbar unerlässliche Angaben, wie etwa das angestrebte Amt fehlen. Zudem zeigt die von Mouritsen dokumentierte ungleiche Verteilung, dass nicht bei allen Kandidaten die Wahlwerbung flächendeckend angebracht wurde. ${ }^{816}$ Das wäre aber nötig gewesen, wenn es sich um die einzige Möglichkeit zur Bekanntgabe der Kandidatur gehandelt hätte. Im Vorfeld der Wahlen war daher die an die Passanten

813 Besonders in Plaut. Rud. 1294-1296 (2); Prop. 3,23.23-24 (4); Plut. Tiberius Gracchus 8,10 (9) und Plut. Pompeius 27.4-5 (10).

814 Besonders in Plin. epist. 8,8,7 (5); Plut. de curiositate (520D-E) (7); Mart. 12,61,7-11 (12) und Lukian, Dial. Mer./Het. dial. 10,4 (14).

815 Vermutlich mit Hilfe von tabulae dealbatae.

816 Mouritsen 1988, 47-59. 
und Anwohner gerichtete Ansprache und der Appell zur Unterstützung das Hauptanliegen der Wahlaufrufe. Da die Aufrufe der jeweiligen Kandidaten sich in jeweils unterschiedlichen Bereichen konzentrierten, ist aber davon auszugehen, dass man vor allem Personen ansprechen wollte, die bereits mit dem Kandidaten in Verbindung standen. Es ging somit zu einem Großteil auch darum, bestehende soziale Beziehungen $\mathrm{zu}$ betonen und zu kräftigen..$^{817}$

Bei den edicta munerum zeigt sich besonders deutlich, dass ein Bedeutungswandel stattfand. Vor den Spielen war es natürlich wichtig, auf Ort, Zeit und Inhalte der geplanten Spiele hinzuweisen. Besonders prominent und groß schrieb man jedoch die Namen der Spielgeber und gegebenenfalls die Anlässe. Dies waren die Aspekte die über den Tag der Veranstaltung hinaus in Erinnerung bleiben sollten. Aus den Ankündigungen von Gladiatorenspielen wurden so dauerhafte Stifterinschriften, die im öffentlichen Raum präsent waren. ${ }^{818}$ Dieses Anliegen spiegelt sich in der Gestaltung ebenso wieder wie in der Vergesellschaftung mit später hinzugekommenen programmata der ehemaligen Spielgeber, die sich auf die Wohltaten für das Volk berufen konnten. Der Aspekt der mittel- und langfristigen Präsenz des Namens spielte auch bei den Wahlaufrufen eine große Rolle - aber auch bei den Grüßen. Diese sind zwar in der Regel an konkrete Personen gerichtet, aber bei einigen, wie etwa dem Kaiser, konnten die Schreiber und Auftraggeber nicht hoffen, dass diese sie jemals lesen würden. ${ }^{819}$ Nur dreimal werden in diesen Grüßen auch Absender genannt, während es sich bei den Gegrüßten in den Dipinti um hochrangige Personen und Kollektive handelt. Im Gemälde in der Casa della Rissa nell'Anfiteatro sind es zudem zwei Grüße, die als Dipinti in die Darstellung aufgenommen wurden. Eine wichtige Funktion dieser Grüße war selbstredend die Ehrung der genannten Adressaten. Insofern sind sie mit Statuenstiftungen auf Platzanlagen und in öffentlichen Gebäuden vergleichbar. Diese dienten jedoch zu einem großen Teil auch dazu, die Loyalität des Stifters, dessen Freigebigkeit und Zustimmung zum Empfänger der Ehrung auszudrücken. ${ }^{820}$ Da bei den Glückwunsch-Dipinti nur selten der Stifter bzw. der Grüßende genannt wird, scheint dieser Aspekt hier eine wesentlich geringere Rolle gespielt zu haben. Vielmehr spricht vieles dafür, dass solche Grüße die Präsenz des Gegrüßten evozieren sollten: einerseits, um an vergangene vorbildhafte Leistungen und Ereignisse zu erinnern, deren Tatkraft und herausragende Stellung vor Augen zu führen, andererseits aber auch, um bestimmte Atmosphären zu erzeugen, die den Straßenraum auch gegenüber den loci celeberrimi der Platzanlagen aufwerteten.

817 So auch Mouritsen 1988, 68-69.

818 Auch Dipinti, die bereits von 200 Jahren ausgegraben wurden, sind heute zum Teil noch gut erhalten.

$819 \mathrm{Zu}$ an den Kaiser oder mit diesem verbundene Personen(gruppen) gerichteten Grüßen: vgl. CIL IV 528 (Dipinto). 820a (Graffito). 1074 (Dipinto). 1612 (Graffito). 2460 (Graffito). Grüße an große Gruppen, wie etwa die Nolani: CIL IV 1512 (Graffito). 4262 (Graffito).

820 Und dies besonders auch dann, wenn der Kaiser die Statue sehr wahrscheinlich nie zu Gesicht bekommen würde. Vgl. von den Hoff 2011, 17. 41-42. 
Bei vielen der Dipinti konnte schließlich eine enge Verknüpfung mit der Semantik des jeweiligen Kontextes und Raumes festgestellt werden. In der Nähe des Amphitheaters kamen vermehrt edicta munerum vor. In den Nekropolen gab es nicht nur Grabinschriften, sondern auch Dipinti, Graffiti und Steininschriften, die auf deren Charakter als Grenz- und Zwischenraum im Verhältnis zum Stadtinneren und den Nachbarstädten Bezug nahmen und diesen somit verstärkten. Bei Steininschriften, die an gestifteten Gebäuden und Ehrenmonumenten, an räumlichen Grenzen und an Gräbern abgebracht wurden, sind solche Verknüpfungen besonders evident. Diese trugen unmittelbar zur Bedeutungsgenese bei bzw. sind selbst nur an einem bestimmten Ort und eingebunden in bestimmte Strukturen zu verstehen. Für manche städtische Teilräume, wie das forum, das Theater, das Amphitheater oder Thermen ist es evident, dass diesen vielfältige, teilweise sehr genau bestimmbare Funktionen und Eigenschaften zugewiesen wurden. In den Fallbeispielen zeigte sich aber, dass auch an kleineren Teilbereichen des Straßenraumes „special places“821 für die Nutzer und Anwohner entstanden und gestaltet wurden. Auch dort boten sich somit Rahmenbedingungen für die Anbringung und Wahrnehmung der Wandinschriften, die weit über die Sichtbarkeit hinausgingen.

\subsection{Korrelationen von Form und Inhalt}

In den vorangegangenen Kapiteln wird deutlich, dass die Inschriften nicht als Verschriftlichung mündlicher Rede zu verstehen sind. Für Ihre Gestaltung galten spezifische Konventionen und Regeln. Dies gilt für Steininschriften ebenso wie für die Dipinti und die Graffiti, wobei auch hier je nach Gattung und Inhalt zu unterscheiden ist. Kruschwitz konnte z. B. zeigen, dass in den Versgraffiti sehr wohl bei der Textverteilung auf die metrische Struktur Rücksicht genommen wurde. ${ }^{822}$ Im Überblick über die verschiedenen Inschriften stellte sich heraus, dass Form und Inhalt korreliert waren und mit Blick auf Produktion wie Rezeption wechselseitig konstitutiv waren. Für die einzelnen Inschriften und soweit möglich für Inschriftengruppen ist dabei zu fragen, ob und wie bestimmte Formen bestimmten Inhalten zugeordnet waren und wie sich dies auf die Gestaltung und die Wahrnehmung auswirkte. An welchen Stellen lassen sich Brüche zwischen Sprache und Schrift erkennen, wo eine besondere Nähe? Wie wirkte sich eine formalisierte Gestaltung auf das Verhältnis von Materialität und Medialität der Inschriften aus?

Besonders bei den Steininschriften führte der Umstand, dass es sich um offizielle oder offiziell genehmigte Inschriften handelte, zu einheitlichen Gestaltungsformen. Das wird z.B. an den kaiserzeitlichen Monumenten am forum deutlich, bei denen

821 Vgl. Kapitel 1.4.1.

822 Vgl. Kruschwitz 2008, 240-241. 
großer Wert auf ein ebenmäßiges und einheitliches Schriftbild gelegt wurde. ${ }^{823}$ Aber auch bei Steininschriften nutzte man verschiedene Schriftarten und Gestaltungsvarianten. Bei Grabinschriften kommen serifenlose Formen ebenso wie die actuaria und die capitalis monumentalis vor. Bei Graffitoinschriften ließ sich keine Korrelation zwischen Form und Inhalt feststellen, abgesehen davon, dass bestimmte Textsorten nur in Form von Graffiti vorkommen. Bei den Dipinti konnte dagegen festgestellt werden, dass besonders für die edicta munerum und die programmata sich Formen und Konventionen entwickelt hatten, die offenbar von zahlreichen Schreibern und unabhängig von der Person des Auftraggebers angewendet wurden.

Bei den programmata wurde die actuaria verwendet. Bestimmte Abkürzungen wurden regelmäßig eingesetzt und teilweise zu nexus verschmolzen. Man malte mit roter oder schwarzer Farbe und verteilte den Text meist auf zwei Zeilen dicht aneinandergesetzter Buchstaben. Gerade die Verwendung von Abkürzungen ermöglichte eine schnelle Anfertigung unter Einsatz formelartiger Wendungen und die Kombination von auch inhaltlich standardisierten Bausteinen. Die Abkürzungen selbst hatten mit Sicherheit keine direkte lautliche Entsprechung, sondern mussten vom Leser mit umfangreichen Vorkenntnissen korrekt erkannt und entschlüsselt werden. ${ }^{824}$ All diese Gestaltungsmerkmale ermöglichten es, den jeweiligen Dipinto rasch wahrzunehmen und grob inhaltlich einzuordnen. ${ }^{825}$ Den Schreibern ermöglichte es, auch mit wenigen Angaben und in großer Geschwindigkeit zahlreiche Wahlaufrufe für den jeweiligen Kandidaten anzubringen. ${ }^{826}$ Dass sehr zügig gearbeitet wurde, kann an einigen der programmata gut beobachtet werden. ${ }^{827}$ Die Gründe, warum die pompejanischen Wahlaufrufe einander alle ähneln, wo es doch aus heutiger Sicht darum geht, sich von anderen abzusetzen und sich selbst bzw. die politische Partei als Marke zu kreieren, sind mehrgestaltig. ${ }^{828}$ Auf der einen Seite waren die programmata nur ein Teil des Bewerbungsverfahrens. Wichtiger war vermutlich die persönliche Beziehungspflege

823 Vgl. CIL X 788. 790. 791.

824 Vgl. zu diesem Aspekt: Krämer 2006, 76. Dazu, allerdings mit Bezug auf Abkürzungen in Steininschriften: Susini 1989, 297.

825 Vgl. Kapitel 2.2.2.4. Zwar lassen sich wichtige Punkte feststellen, in denen die gesprochene Sprache und insbesondere die Aussprache direkt auf diese Schriftzeugnisse einwirkt, wie Rex E. Wallace herausgearbeitet hat (Wallace 2005, XXIV-XXXIX). Wallace geht dabei besonders auf die Längen ein, zu deren Darstellung apices verwendet wurden. Viele gestalterische Elemente finden Entsprechungen in der gesprochenen Sprache: Wahl des Akkusativs in den Fällen, in denen der Name ausgeschrieben ist, Orientierung der Zeilen am üblichen Satzbau. Dagegen können weder die unterschiedlichen Zeilenhöhen und das blockhafte Arrangement der Texte mit der Linearität gesprochener Sprache in Verbindung gebracht werden. Vgl. vor allem: Krämer 2003, 157-166; Krämer 2006, 76-77.

826 Ausnahmen bestätigen hier die Regel. Vgl. 4.1.3.2 und 5.1.2.

$\mathbf{8 2 7}$ Vgl. Kapitel 2.2.2.3.1.

828 Vgl. zur Wirkungweise und Gestaltung moderner Wahlplakate: Geise/Brettschneider 2010, 77-91. Geise und Brettschneider stellen Bilder als wichtigsten Bestandteil heraus, um langfristige Aufmerksamkeit zu erzielen, welche in den programmata überhaupt keine Rolle spielen. 
wie auch das Gewinnen von Stimmen durch Wohltaten wie z. B. Gladiatorenkämpfe. Auf der anderen Seite lag in der Gleichförmigkeit auch eine Tugend. Diejenigen Kandidaten, deren Wahlaufrufe den formalen Konventionen entsprachen, konnten es sich offenbar leisten, professionelle Schreiber zu beauftragen und wussten um die Regeln und Gepflogenheiten. Nicht Individualität war bei den Kandidaten um die städtischen Ämter gefragt, sondern Konformität.

Bei den kaiserzeitlichen edicta munerum ging es dagegen weniger um Masse als um eine hochwertige Ausführung und monumentale Wirkung der einzelnen Inschrift. Dieser sollte sehr wohl unter den benachbarten Schriftzügen an der jeweiligen Wand herausstechen, wie in den Fallbeispielen deutlich wurde. Auch bei diesen Veranstaltungsankündigungen konnte festgestellt werden, dass sie nach einem bestimmten Muster angelegt wurden. Die erste Zeile war sehr groß in vergleichsweise breiten und sehr sorgfältig gestalteten Buchstaben geschrieben und beinhaltete meist den Namen des Veranstalters oder den Anlass für die Spiele, ${ }^{829}$ während die Details, die für die potentiellen Besucher selbstredend den höheren Informationswert hatten, deutlich kleiner darunter gesetzt wurden. Die erste Zeile diente einerseits die Inszenierung und Glorifizierung des Veranstalters, der sich wie der Stifter eines Gebäudes selbstbewusst nennen konnte, ohne dabei wie bei einer Architekturstiftung an das Bauwerk selbst gebunden zu sein. Andererseits bildete die erste Zeile ein Erkennungsmerkmal dieser Inschriften und stellte zugleich einen Blickfang dar, der auch einen flüchtigen Betrachter darauf hinweisen konnte, um welche Art von Inschrift es sich handelte und welche Informationen sich in den folgenden Zeilen verbargen. Wie oben erläutert verloren langfristig die unteren Zeilen an Bedeutung. Nachdem die Spiele stattgefunden hatten, gewann dafür der Aspekt der Memorialisierung und Ehrung eine umso höhere Signifikanz.

Sowohl bei den programmata als auch bei den edicta munerum wurde somit die Form zugleich auch zum Sinnbild für den Inhalt, sodass der Betrachter oder der eilige Passant die Wandinschriften grob einordnen konnte, bevor er seine Aufmerksamkeit bewusst darauf richtete oder sie las. ${ }^{830}$ Aus Sicht des Rezipienten konnte also auch die Form zum Inhaltsträger werden. Dies war wichtig in einem Umfeld, in dem nicht nur Wandinschriften, sondern auch Fassadenmalereien und zahlreiche andere visuelle Reize auf den Betrachter einstürmten. Wohl in den seltensten Fällen gingen die Passanten mit dem Ziel durch die Straßen, neue interessante Dipinti ausfindig zu machen und zu lesen. Vielmehr waren die Wahlaufrufe und Veranstaltungsankündigungen etwas, das zusätzlich zu den Eindrücken, die unmittelbar relevant waren, wie Verkehrsaufkommen, Hindernisse, Zurufe, bekannte Gesichter etc., vorhanden war.

829 Bei einigen edicta munerum wurde hingegen die Gladiatorenanzahl in der ersten Zeile genannt, z. B.: CIL IV 9983a für Spiele in Cumae. Hier fehlte dagegen eine Nennung des Veranstalters oder des Anlasses.

830 Dazu ausführlich: Kapitel 2.2.2.4. Vgl. die Ergebnisse von Kruschwitz/Campbell 2009. 
In dieser Situation war es wichtig, dass eine möglichst einfache und schnelle Einordnung möglich war.

In Fällen wie den programmata des Fufidius, in denen das übliche Erscheinungsbild nicht eingehalten wurde, konnten sich daher auf Seiten der Rezipienten auf unterschiedlichen Ebenen verschiedene Effekte ergeben. Einerseits wurden diese Dipinti nicht so schnell der inhaltlichen Gruppe der Wahlaufrufe zugeordnet. Andererseits erregten sie vielleicht aufgrund ihres für Dipinti insgesamt eher ungewöhnlichen Aussehens mehr Aufmerksamkeit. Im zweiten Schritt stellte sich für den nun bewusst lesenden Betrachter die Frage, ob es sich tatsächlich um ernst zu nehmende programmata handeln konnte. Wo bei den meisten Texten dieser Art davon auszugehen ist, dass sie in Absprache und im Einvernehmen mit dem Kandidaten durch professionelle Schreiber ausgeführt wurden, entsteht hier der Eindruck, dass Fufidius gänzlich in Eigenregie agierte. In einem solchen Fall hing die Wirkung stark vom persönlichen Standing des Urhebers ab.

In Fällen, in denen die Form gegenüber dem Text und dem Inhalt so stark in den Vordergrund tritt, dass ein Inhalt nur noch schwierig zu erkennen ist, gewinnt auch bei Inschriften die Materialität gegenüber der Medialität der Schrift noch stärker an Einfluss. Dabei kann es sich wie bei vielen Graffiti um schwer zu entziffernde Buchstaben, aber auch um schlecht erhaltene Inschriften handeln. Auch oskische Dipinti, die sprachlich und schriftlich allenfalls für einen Teil der Bevölkerung verständlich waren, „funktionierten“ kaum noch als Medien. Hinzu kommen gerade unter den Graffiti solche, die nur aus einem oder wenigen Buchstaben bestehen und als Texte gar nicht verständlich sind. Ebenso gewinnt die Materialität auch für den Betrachter an Bedeutung, wenn sich der Urheber an besonderen und außergewöhnlichen Gestaltungen versuchte. ${ }^{831}$ Die Häufung und hohe Anzahl der Dipinti konnte allerdings auch ein Faktor dafür sein, dass diese in einem Kontext nicht als Texte, sondern vorrangig ästhetisch rezipiert wurden. Dadurch, dass sie an den Wänden mit Gemälden vergesellschaftet waren und notgedrungen Einfluss auf das Aussehen der Fassade nahmen, wurden sie Teil der Gestaltung des öffentlichen Raumes.

\subsection{Kontext, Handlung und Wahrnehmung von Inschriften}

Sowohl in Pompeji als auch in Herculaneum wurden durch den Ausbruch des Vesuv Schriftzeugnisse in unvergleichlicher Menge und ansonsten selten zu findenden Materialien konserviert. Beide Städte zeichnen sich daher heute durch ein Nebeneinander von außerordentlich zahlreichen Schriftzeugnisse aus. Dabei fallen grundsätzliche Unterschiede auf, die zum Teil durch die verschiedenen Bedingungen bei der Verschüttung und die Dokumentation der Funde zu erklären sind. Allerdings wird an den

831 Vgl. die besonders gestalteten Graffiti in Kapitel 3.2.4. Vgl. Lohmann 2018, 260-263. 
erhaltenen Zeugnissen auch deutlich, dass die Einwohner oder Besucher der beiden Städte sich bereits in der Antike nicht demselben Spektrum an Schriftlichkeit gegenübersahen, sondern dass lokale Spezifika sich auch in diesem Bereich niederschlugen. ${ }^{832}$ In verkürzter Form kann festgehalten werden, dass in Herculaneum vor allem steinerne Ehren- und Stifterinschriften zu sehen waren, die sich um die öffentlichen Gebäude herum konzentrierten. In Pompeji treten zu diesen Tausende von Graffiti und Dipinti hinzu, wobei insbesondere die Dipinti optisch sehr präsent waren und heute noch sind..$^{83}$

Die verschiedenen Inschriftengattungen waren nicht alle an den gleichen Stellen in gleichem Maße präsent. Während die Steininschriften vor allem an dafür offiziell vorgesehenen oder sogar reservierten Stellen angebracht waren, scheint bei Graffiti und Dipinti genau das Gegenteil der Fall zu sein. Auf den ersten Blick scheinen sie sich überall und besonders an den am stärksten frequentierten Durchgangsstraßen zu befinden. Die Platzierung der programmata, edicta munerum und der inhaltlich ganz unterschiedlichen Graffiti war jedoch ebenfalls nicht beliebig. Wichtige Faktoren waren dabei Präsenz und Sichtbarkeit. Doch wie im vorangehenden Abschnitt klar wurde, spielten darüber hinaus auch das Verhältnis zu dauerhaft in der Umgebung vorhandenen Elementen, persönliche Beziehungen, die Präsenz anderer Inschriften im direkten und weiteren Umfeld, Gewohnheiten von Menschen und individuelle strategische Überlegungen eine große Rolle. ${ }^{834}$ Daher kann von Beliebigkeit keine Rede sein, sondern vielmehr von einer solchen Komplexität, dass theoretisch jede Inschrift als Einzelfall ausführlich untersucht werden müsste, um die Faktoren und Überlegungen, die zur Wahl ihrer Anbringungsstelle führten, näher zu bestimmen.

Dennoch waren auch für einen Besucher der Stadt, der von außerhalb kam, gewisse Regelmäßigkeiten zu erkennen. Dies betrifft zum einen die Steininschriften, denen er bereits begegnete, bevor er die Stadt betreten hatte. In den Nekropolen durfte

832 Wie Wallace-Hadrill betont, sollte allein das schon Grund genug sein, den Befund in Pompeji nicht als Modell auf die „römische Stadt“ im Allgemeinen zu übertragen: Wallace-Hadrill 2011a, 296297.

833 Solin betont aber auch, dass Pompeji kein Einzelfall ist und dass z. B. in Ostia ebenfalls eine lebendige Graffiti-Kultur belegt ist: Solin 1973a, 101. Auf der Grundlage der bis 1973 bekannt gewordenen Zeugnisse aus Herculaneum beschrieb Solin die herculanensischen Graffiti als enttäuschend: Solin 1973a, 97. 98: „Die wenigen Texte, die Verse, Sentenzen oder irgendwelche Maximen aufweisen oder überhaupt ein Verhältnis zur literarischen Kultur widerspiegeln, scheinen fast ausschliesslich von den dort ansässigen Römern oder von dort geweilt habenden Touristen zu stammen." Die Textkultur der jeweiligen Stadt sei „durch ihre gesellschaftliche Struktur und die wirtschaftlichen Verhältnisse bestimmt“. Er erklärt die festgestellten Differenzen mit „der unterschiedlichen Atmosphäre der Stadt sowie der unterschiedlichen Veranlagung und Einstellung der Stadtbewohner zu Fragen des alltäglichen Lebens auf all seinen Stufen“ (Solin 1973a, 101-102).

834 Besonders die verschiedenen Strategien bei der Anbringung der programmata werden von Mouritsen ausführlich behandelt: Mouritsen 1988, 47-60. Zu den edicta munerum: Sabbatini Tumolesi 1980, 119-122. 
er zahlreiche Grab-tituli erwarten, sah sich jedoch zugleich vielen gemalten Inschriften und Graffiti gegenüber, die damals noch deutlich besser zu sehen gewesen sein müssen als heute. Diese waren auf dieselben Grabfassaden geschrieben, an denen auch die tituli angebracht waren. Wenn er das Tor durchschritten hatte und auf seinem Weg durch die Stadt auf den breiten Hauptverkehrsachsen blieb, dürfte er zunächst nur noch sehr wenige Steininschriften gesehen haben, dafür jedoch vor allem Dipinti, die teilweise bis zur Unleserlichkeit an den Fassaden übereinander geschrieben waren. Doch auch in den Seitenstraßen begegneten ihm an verschiedenen Stellen immer wieder einzelne oder gruppierte Dipinti. Sobald er das forum erreicht hatte oder eines der öffentlichen Gebäude betrat, herrschten wieder die Steininschriften vor. Die oft sehr kleinteiligen Graffiti dagegen waren ihm vermutlich nur dann aufgefallen, wenn sie sich zu größeren Ansammlungen verbanden, oder aber, wenn er sehr nahe an einer mit Graffiti beschriebenen Wand entlangging. Ansonsten waren sie eher nicht dazu angetan, die Blicke von gänzlich unvoreingenommenen Betrachtern anzuziehen.

Inschriften waren nicht die einzigen Artefakte und Bestandteile des öffentlichen Raumes, denen sich der Besucher gegenübersah. Verschiedene Vorrichtungen wie Laufbrunnen oder kleine Altäre, Fassadenbilder, bauliche Strukturen und je nach Tageszeit temporäre Aufbauten von Verkaufsständen begegneten ihm im öffentlichen Raum der Stadt. Insbesondere sah er sich - ebenfalls abhängig von der Tageszeit auch Menschenmengen oder einzelnen Einwohnern gegenüber oder wurde vielleicht im Vorbeigehen auf das Innenleben einer taberna aufmerksam. Die Inschriften waren ein Teil dieser vielen Elemente, die im öffentlichen Raum ständig oder vorübergehend präsent waren. Die verschiedenen Inschriftengattungen, die sich zum einen vom Material her und zum anderen inhaltlich umschreiben lassen, waren jedoch wiederum in unterschiedliche Handlungszusammenhänge eingebunden, sowohl im Rahmen der Herstellung als auch im Rahmen der Wahrnehmung. Das bezieht sich sowohl auf ihre Verknüpfung mit Bewegungsabläufen und ihrer Position innerhalb des Stadtgebietes, als auch ganz konkret auf die Sichtbarkeit und Lesbarkeit. Aus dieser Perspektive erscheint der Inhalt der Inschriften, der sonst meist das wichtigste Beschreibungskriterium von Inschriften ist, als ein Aspekt unter mehreren. Besonders bei Steininschriften sind Inhalt und Aufstellungsort auf das engste korreliert, während bei Graffiti gerade das Nebeneinander und Zusammenspiel von verschiedenen Inhalten eine große Rolle spielt.

Dabei ist an Wahrnehmungsweisen zu denken, wie sie Paul Zanker mit Bezug auf Bildräume im kaiserzeitlichen Rom beschreibt, wenn er von dem unaufmerksamen Betrachter und gewohnheitsmäßigen Passanten annimmt, dass er, ohne dass ,er sich je Gedanken über das Programm eines der Bögen gemacht hätte, eine bestimmte Vorstellung von einem Ehrenbogen und von den Bildschemata der siegreichen Kaiser, deren Siegesgespanne auf den Bögen standen“, mit sich trug. ${ }^{835}$ Ein wichtiger Unter-

835 Vgl. Zanker 2000, 223. 
schied zu den Ehrenbögen in Rom besteht natürlich darin, dass in Pompeji monatlich, möglicherweise wöchentlich oder täglich neue Inschriften hinzukamen und dass jährlich kurz vor den Wahlen auf einen Schlag etliche neue Dipinti Teil des öffentlichen Raumes wurden. Der Betrachter hatte somit die einzelne Inschrift noch nicht seit Jahren oder Jahrzehnten gesehen und als selbstverständlich in das Straßenbild integriert. Gerade wegen der deutlichen Typenhaftigkeit muss jedoch davon ausgegangen werden, dass auch nur ein flüchtiger Blick oder allein die Wahrnehmung einzelner Merkmale wie etwa Farbe, Anbringungsstelle und Spektrum der Buchstabengrößen dem Einwohner Pompejis ausreichten, um ein neues programma als solches zu erkennen.

Diese Überlegungen ließen sich auch an anderen Schriftzeugnissen in Pompeji und an anderen Orten erproben. Sie zeigen, dass Sinnzuschreibungen auch gänzlich ohne das Lesen der einzelnen Inschrift möglich sind, allein auf der Basis der Materialität und aufgrund der Sehgewohnheiten, die mit jedem neuen derartigen Vorgang der Rezeption aktualisiert werden. Der Aspekt, der hier in den Vordergrund gestellt wird, ist gerade nicht die vermeintliche Linearität und Sprachbezogenheit von Texten und auch nicht eine potentielle Ikonizität von Schrift im Sinne eines Verweisens auf ein Objekt. ${ }^{836}$ Vielmehr greift hier der Begriff der Schriftbildlichkeit, welcher den Text in seiner Materialität in den Mittelpunkt stellt. ${ }^{837}$ Darüberhinausgehend sollen hier jedoch Texte nicht nur als zweidimensionale Phänomene, die Bezüge zwischen ihren Bestandteilen aufweisen, verstanden werden, sondern als dreidimensionale Artefakte, die in einem Raum verankert sind und sich somit immer auch auf diesen beziehen, weil sie nur darin existieren. Dreidimensionalität meint dabei sowohl die teilweise nur schwer beschreibbare Plastizität der Schrift selbst - den Keilschnitt, den Farbauftrag, die geritzten Linien - als auch die Verortung der Inschrift in einem dreidimensionalen Raum.

Allein aufgrund ihrer Masse, vor allem in Pompeji, ist nicht nur der Raum als konstitutiv für die Inschriften anzusehen, sondern umgekehrt müssen auch die Inschriften als signifikanter Bestandteil des öffentlichen Raumes angesehen werden. Dabei ist nicht nur an die optische Wirkung leuchtend roter Dipinti zu denken, sondern auch an das Potential von Graffiti, einen Teilbereich des öffentlichen Raumes dauerhaft zu einem Ort privater Kommunikation zu machen, oder an die Rolle, die Steininschriften bei der Auszeichnung und Hierarchisierung von Ehrenmonumenten spielten, ganz zu schweigen vom performativen Potential der cippi, die einen wichtigen Teilraum markierten und konstituierten. Selbstverständlich ist nicht davon auszugehen, dass die Einwohner oder Besucher von Pompeji oder Herculaneum einen derartig dezidierten Fokus auf diese Inschriften in ihren Städten legten. Viele Inschriften blieben sicherlich von vielen unbemerkt oder gingen in der Vielfalt unter. Dennoch waren sie, sobald sie präsent waren, Teile des städtischen Raumes, so wie verschiedenste andere

836 Vgl. Schmauks 2008, 309-310.

837 Vgl. Krämer 2003. 
Artefakte auch. Während diese Perspektive für Steininschriften bereits lange etabliert ist, konnte hier besonders der Status von Dipinti und Graffiti innerhalb dieses Gefüges neu bestimmt werden. Ein besonders wichtiger Aspekt ist dabei die Rolle, die auch die Dipinti im Rahmen gesellschaftlicher Mechanismen von Selbstdarstellung und Öffentlichkeit spielten.

\subsection{Zusammenfassung oder: Raumaneignung durch Schrift?}

Die Inschriften in Pompeji und Herculaneum treten dem Touristen und Archäologen heute an vielen Wänden und Monumenten der beiden Städte entgegen. Die Buchstaben sind uns vertraut, der Humor und die Themen der Bilder und Texte lassen uns noch heute schmunzeln oder machen uns nachdenklich. Viele der Inschriften lassen uns die Stadt lebendiger erscheinen, als dies leere Häuser, aufgeräumte Straßen, halb zerstörte Gebäude und in Museen aufbewahrtes Fundmaterial möglich machen. Die Ankündigung von Gladiatorenkämpfen oder die Aufrufe zur Wahl nennen uns Akteure des gesellschaftlichen Lebens und füllen in unserer Vorstellung das Amphitheater und die Amtsgebäude am forum mit Leben. In die Wände geritzte Namen und Grüße lassen uns über die Hausbewohner, ihre Freunde und Tätigkeiten spekulieren und führen uns in kleine Gassen und Winkel der Stadt, die für den heutigen Flaneur sonst nur wenig zu bieten haben. Abgesehen von den heute noch an den Wänden sichtbaren Inschriften und den prominent am forum ausgestellten Statuensockeln, sind zahlreiche Inschriften jedoch nur in ihrem Wortlaut bekannt und bieten sich dem Besucher der Ruinen nicht als Teil des öffentlichen Raumes dar. In dieser Arbeit wurde der Versuch unternommen, die pompejanischen Inschriften neu zu bewerten und insbesondere Fragen nach Ihrer Entstehung, ihrer Wahrnehmung und ihrer Bedeutung jenseits ihres Quellenwertes zu den in den Texten verhandelten Sachverhalten $\mathrm{zu}$ beantworten.

Am Anfang steht eine Beschreibung des Materials hinsichtlich der textuellen Inhalte der Inschriften, ihrer Gestaltung und Machart sowie ihrer Verteilung im Stadtgebiet. Daran schließt sich eine Auswertung antiker Schriftzeugnisse zu Inschriften an, die insbesondere Bewertungen seitens der Zeitgenossen aber auch Hinweise auf Schreiber, Leser und die Produktions- wie Rezeptionsvorgänge beinhalten. Im dritten Kapitel des Hauptteils wurden schließlich ausgewählte Teilräume analysiert, um die dortigen Inschriften in ihren räumlichen Kontext einzuordnen und als Teile derselben $\mathrm{zu}$ verstehen. Problematisch sind dabei insgesamt der meist schlechte Erhaltungszustand und die spärliche Dokumentation im CIL. Die Beobachtungen und Resultate werden jeweils am Ende der Kapitel als Zwischenergebnisse zusammengefasst, und sollen hier nicht im Einzelnen wiederholt werden. Im Folgenden seien stattdessen die Ergebnisse, die sich aus der Synthese aller drei Analyseebenen ergeben, noch einmal zusammengeführt. 
Durch die verschiedenen Inschriften treten größere Teile der Bevölkerung als handelnde Individuen in Erscheinung, als es auf andere Arten möglich wäre. Zugleich zeigt sich, dass nicht nur bestimmte Teilräume wie Theater, Nekropolen und ähnliche prominente öffentliche Gebäude und städtische Teilbereiche Orte des öffentlichen Diskurses waren, sondern dass gesellschaftliche Beziehungen und soziales Handeln auch im Straßenraum und an Fassaden und Wänden verhandelt wurden. Die kommunikativen Funktionen der Inschriften waren nicht statisch, sondern veränderten sich im Laufe der Zeit. Während im Zuge der Produktion vor allem die Intentionen des oder der Urheber im Zentrum stehen, war die Rezeption schon unmittelbar nach der Anbringung und im Laufe der Zeit immer weniger von deren Absichten und Vorstellungen abhängig. Vielmehr ergeben sich im städtischen Raum immer wieder neue Sinnzusammenhänge.

Die Vielschichtigkeit und die Möglichkeiten unterschiedlicher Bedeutungszuschreibungen wurden gezielt genutzt. Wandinschriften stellen sich als eine Form der Memorialisierung und Ehrung heraus, die gar nicht so ephemer ist, wie man glauben könnte. Dadurch dass Beschriftungen im Straßenraum weniger reguliert und weniger exklusiv waren als etwa die Option, ein Monument auf dem forum zu erhalten, können die Dipinti und Graffiti sogar langfristiger zu sehen gewesen sein als die Ehrenstatuen, die nach einiger Zeit wieder abgeräumt oder an einen anderen Ort versetzt werden konnten. Form und Inhalt der Inschriften sind notgedrungen verknüpft, da das eine nicht ohne das andere existiert. Besonders bei den programmata und den edicta munerum wurde aber durch Konventionalisierung in der Kaiserzeit die Form so sehr zum Wiedererkennungsfaktor, dass eine inhaltliche Zuordnung stattfinden konnte, ohne dass sie im eigentlichen Sinne gelesen wurden.

Die konkrete Wahrnehmung aber auch die Frage, ob eine Inschrift überhaupt an einer bestimmten Stelle angebracht werden konnte und sollte, war abhängig auch vom Umfeld und dessen Bestandteilen, weiteren Inschriften, Handlungen und Tätigkeiten des Betrachters, dessen Prädispositionen sowie dessen Erwartungshaltung. Inschriften ihrerseits wirkten ästhetisch und semantisch auf den Raum, in dem sie sich befanden. Besonders deutlich wurde dies bei den Wahlaufrufen für auswärtige Kandidaten in der Nekropole vor der Porta Nocera oder in Veranstaltungsankündigungen in der Nähe des Amphitheaters. Die Signifikanz des Kontextes zeigte sich aber auch in den unterschiedlichen Strategien, Wahlaufrufe möglichst gut sichtbar und günstig zu inszenieren.

Die Inschriften wirkten aber auch durch ihre schiere Präsenz auf den Raum und auf das Handeln der anderen Akteure. Indem eine Inschrift oder ein Monument aufgestellt wurde, ein Dipinto an eine Wand gemalt oder ein Graffito an eine bestimmte Stelle geschrieben oder gezeichnet wurde, wurde dieser Ort besetzt. So entstanden neue Sinnbezüge, in der eine vormals weiße Wandfläche oder eine ehemals leere Fläche auf dem forum einen neuen Stellenwert erhielt. Durch die Anbringung von Dipinti und Graffiti konnten Akteure aus unterschiedlichen Bevölkerungsgruppen auch den öffentlichen Raum modifizieren und für sich oder ihre Belange beanspruchen. Das 
forum war offenbar gezielt von dieser Praxis ausgenommen worden. Hier finden sich nur Stifter-, Bau-, Weih- und vor allem Ehreninschriften. Auch dabei handelt es sich um eine Form der Aneignung, die allerdings hochgradig reguliert war und folglich durch die Materialität und Präsenz der Inschriften die zugrundeliegende Ordnung immer wieder reproduzierte und aktualisierte.

Aneignung bedeutet in diesem Zusammenhang nicht, dass ein Teilbereich des öffentlichen Raumes der allgemeinen Nutzung entzogen worden wäre. ${ }^{838}$ Es bedeutet auch nicht lediglich, dass die Bürger Pompejis einem dem Menschen eigenen, universellen Wunsch nach Verewigung nachgekommen wären. Vielmehr lassen sich in den Inschriften verschiedene Formen der Inanspruchnahme des öffentlichen Raumes greifen. Die Schreiber hinterlassen die Inschriften als Spuren ihrer selbst. Durch die Nennung von Personen werden diese mit Orten verknüpft. Besonders eindrücklich ist dies etwa bei Grüßen an den Kaiser oder bei den cippi des Suedius Clemens, durch welche Räume definiert und fest mit bestimmten Vorstellungen, Ereignissen und Ordnungen verknüpft. Aber auch mit so bescheidenen Inschriften wie den tituli memoriales eigneten sich die Akteure den öffentlichen Raum an. Successus hic ${ }^{839}$ bezeugt weniger den Absicht, sich für eine ferne oder nahe Zukunft zu verewigen, sondern vielmehr den Wunsch, in der damaligen Gegenwart am öffentlichen Leben teilzuhaben und den eigenen Aktivitäten einen sichtbaren Stellenwert zu verleihen.

838 Wie z. B. bei der Bestuhlung von Gehwegen und Platzanlagen durch Cafés. 839 CIL IV 5305. 
